\title{
A DUALITY BETWEEN SPHERES AND SPHERES WITH ARCS
}

\author{
CARL D. SIKKEMA ${ }^{1}$
}

Abstract. The purpose of this paper is to extend the authors duality between nearly tame spheres and nearly tame arcs to a duality between wild spheres and tame spheres with nearly tame arcs properly attached, one arc for each wild point of the wild sphere.

1. Let $S^{3}$ be the one point compactification of Euclidean 3-space $R^{3}$ and let $S^{2}$ and $B^{3}$ be the unit 2-sphere and 3-ball in $R^{3}$ centered at the origin, respectively. For $X \subset S^{3}$, let $\mathrm{Cl} X$ denote the closure of $X$ and $V_{\epsilon}(X)$ denote the $\epsilon$-neighborhood of $X$.

A set $X$ in $S^{3}$ is locally flat at a point $x \in X$ if there is a neighborhood $U$ of $x$ and a canonical set $Y$ in $R^{3}$ such that $(U, U \cap X)$ is topologically equivalent to $\left(R^{3}, Y\right)$.

2. Let $\delta$ be the collection of all 2 -spheres in $S^{3}$. Let $\Sigma \in \mathcal{S}$ and let $C_{0}$ and $C_{1}$ be the closed complementary domains of $\Sigma$ in $S^{3}$. By [4] or [5] there are imbeddings $h_{i}: C_{i} \rightarrow S^{3}$ such that $\mathrm{Cl}\left(S^{3}-\left(h_{0}\left(C_{0}\right) \cup h_{1}\left(C_{1}\right)\right)\right)$ is an annulus. Hence there is an imbedding

$$
g: S^{2} \times[0,1] \rightarrow \mathrm{Cl}\left(S^{3}-\left(h_{0}\left(C_{0}\right) \cup h_{1}\left(C_{1}\right)\right)\right)
$$

such that $g\left(S^{2} \times i\right)=h_{i}(\Sigma)$ and $h_{0}^{-1} g(y, 0)=h_{1}^{-1} g(y, 1)$ for each $y \in S^{2}$. Without loss of generality $g\left(y, \frac{1}{2}\right)=y$.

Let $X_{i}$ be the set of points $x$ in $S^{2}$ such that $h_{i}(\Sigma)$ is not locally flat at $g(x, i), i=0,1$, and let $X=X_{0} \cup X_{1}$. Let $a_{x}=g\left(x \times\left[0, \frac{1}{2}\right]\right)$ if $x \in X_{0}-X_{1}$, let $a_{x}=g\left(x \times\left[\frac{1}{2}, 1\right]\right)$ if $x \in X_{1}-X_{0}$ and let $a_{x}=g(x \times[0,1])$ if $x \in X_{0} \cap X_{1}$. It follows from Lemma 6 of [6] that each arc $a_{x}$ is cellular. Then by Lemma 4 of [6] or Theorem 10 of [7] each arc is locally flat except possibly at one end point.

Let $S=S^{2}$. Then $S \cup\left(\cup a_{x}\right)$ satisfies the following properties:

(1) Each arc $a_{x}$ intersects the flat (tame) 2 -sphere $S$ at one point $x$,

(2) Each $a_{x}$ is locally flat (locally tame) except possibly at one end point not on $S$,

Received by the editors November 3, 1969.

AMS Subject Classifications. Primary 5478.

Key Words and Phrases. Arc, 2-sphere, 3-sphere, duality, tame, wild, locally flat, upper semicontinuous decomposition.

${ }^{1}$ This work was partially supported by the National Science Foundation, Grant GP-11943. 
(3) $S \cup\left(\cup a_{x}\right)$ is not locally flat at the end points of the arcs which are not on $S$ but is locally flat at all other points,

(4) If $a_{y}$ is locally flat at an end point $p_{y}$ not on $S$, then $p_{y}$ is a limit point of end points of other arcs in the collection $\left\{a_{x}\right\}$,

(5) $\bigcup a_{x}$ is closed,

(6) $\left\{a_{x}\right\}$ is the set of nondegenerate elements of an upper semicontinuous decomposition $d: S^{3} \rightarrow d\left(S^{3}\right)$ such that $d\left(S^{3}\right)$ is $S^{3}$.

A sphere with arcs is a set $S \cup\left(\cup a_{x}\right)$ which satisfies properties (1)(5). Let $a$ be the collection of spheres with arcs each of which also satisfies property (6). Then we can define a correspondence $\Gamma: \delta \rightarrow Q$ by $\Gamma(\Sigma)=S \cup\left(\cup a_{x}\right)$ as indicated in the preceding paragraphs, although $\Gamma$ is not a well-defined function. However, if $\delta_{*}$ and $a_{*}$ are the collections of equivalence classes of $\delta$ and $\alpha$, respectively, then it is easy to see that $\Gamma$ induces a function $\Gamma_{*}: \delta_{*} \rightarrow Q_{*}$.

Now define $\Psi: Q \rightarrow S$ by $\Psi\left(S \cup\left(\cup a_{x}\right)\right)=d(S)$, where $d$ is the decomposition map of property (6). It is easy to see that $\Psi$ induces a function $\Psi_{*}: Q_{*} \rightarrow S_{*}$.

TheOREM 1. $\Gamma_{*}$ and $\Psi_{*}$ are inverses of each other.

This theorem (to be proved in $\$ 4$ ) establishes the duality between $S$ and $Q$. A dual of $\Sigma \in \Sigma$ is any $S \cup\left(\cup a_{x}\right) \in \Gamma_{*}(\mathcal{S}) \subset Q$ and a dual of $S \cup\left(\cup a_{x}\right) \in Q$ is any $\Sigma \in \Psi_{*}\left(S \cup\left(\cup a_{x}\right)\right) \subset$ s.

Notice that a collection of arcs alone does not determine a unique sphere. In particular, Example 1.2 of Fox and Artin [3] and its reflection can be attached to a tame 2 -sphere so that the dual wild sphere may have both or just one of its complementary domains simply connected.

Properties (3) and (6) are necessary in order to keep $a$ small enough to establish the duality; it is easy to find an example to show that property (3) is necessary and Ball [1] has described a sphere with arcs which fails to satisfy property (6). Properties (4) and (5) insure that an arc $a_{x}$ is in the collection of arcs if and only if $x$ corresponds to a wild point of the dual sphere.

3. In this section we will use polar coordinates, that is, $p=(\theta, r)$ where $r$ is the distance from $p$ to the origin and $\theta$ is the projection (from the origin) of $p$ onto $S^{2}$.

THEOREM 2. If $S \cup\left(\cup a_{x}\right)$ is a sphere with arcs such that $S=S^{2}$ and $\cup a_{x} \subset B^{3}$, then $S^{2}$ has a collar $C$ in $B^{3}$ such that $a_{x} \cap C$ is the collar at $x$. In fact, there is a homeomorphism $h: S^{2} \times[0,2] \rightarrow \rightarrow B^{3}$ such that $h\left(x \times[1,2] \cup a_{x}\right)=a_{x}$ for each $x \in X$. 
Proof. Since $S^{2} \cup\left(\cup a_{x}\right)$ is locally flat, there is a finite open (in $\left.B^{3}\right)$ cover $\left\{C_{1}, \cdots, C_{n}\right\}$ of $S^{2}$ by local collars in $B^{3}$ such that, for $x \in C_{i} \cap X, a_{x} \cap C_{i}$ is the collar at $x$. Let $U_{i}=C_{i} \cup\left(\left(C_{i} \cap S^{2}\right) \times[1,2]\right)$ and let $\left\{V_{1}, \cdots, V_{n}\right\}$ be an open (in $S^{2}$ ) cover of $S^{2}$ such that $\mathrm{Cl} V_{i} \subset U_{i}$. Let $h_{i}$ be a homeomorphism of $S^{2} \times[0,2]$ onto itself such that $h_{i} \mid\left(S^{2} \times[0,2]\right)-U_{i}=1, h_{i}\left(V_{i}\right) \subset$ Int $B^{3}$ and, for $x \in C_{i} \cap X$,

$$
h_{i}\left((x \times[1,2]) \cup a_{x}\right)=(x \times[1,2]) \cup a_{x} .
$$

Let $h=h_{n} \cdots h_{1}$ and let $g$ be a homeomorphism of $S^{2} \times[0,2]$ onto $B^{3}$ such that $g(x, 2)=(x, 1)$ for $x \in S^{2}$ and such that $g h\left((x \times[1,2]) \cup a_{x}\right)$ $=a_{x}$ for $x \in X$. Then $g h$ is the required homeomorphism.

THEOREM 3. If $S \cup\left(\cup a_{x}\right)$ is a sphere with arcs and $\bigcup a_{x}$ is contained in one of the closed complementary domains $B$ of $S$, then $S \cup\left(\cup a_{x}\right)$ satisfies property (6). In fact, if $S=S^{2}$ and $B=B^{3}$, then there is a map $g: S^{2} \times[0,2]$ onto itself such that $g$ maps each $a_{x}$ to a point, $g(x \times[1,2])$ $=(x \times[1,2]) \cup a_{x}$, for $x \in X$, and $g \mid S^{2} \times 2=1$.

Proof. Without loss of generality $S=S^{2}$ and $\bigcup a_{x} \subset B^{3}$. Let $p_{x}$ be the end point of $a_{x}$ not on $S^{2}$. By techniques similar to those in the proof of Theorem 2, we can prove that given a sequence of positive numbers $\left\{\epsilon_{i}\right\}$ there is a sequence of homeomorphisms $\left\{h_{i}\right\}$ of $S^{3}$ onto itself such that

$$
h_{i} \mid S^{3}-V_{\epsilon_{i}}\left(\bigcup a_{x}\right)=h_{i-1}, \quad h_{i}\left((x \times[1,2]) \cup a_{x}\right)=(x \times[1,2]) \cup a_{x}
$$

and

$$
h_{i}\left(\bigcup_{a_{x}}\right) \subset V_{1 / i}\left(\bigcup_{p_{x}}\right) .
$$

By a proper choice of the $\epsilon_{i}$, these homeomorphisms converge to a map $h$ of $S^{3}$ onto itself such that $h \mid S^{3}-\bigcup a_{x}$ is a homeomorphism, $h\left(a_{x}\right)=p_{x}, h(x \times[1,2])=(x \times[1,2]) \cup a_{x}$ and $h \mid S^{3}-S^{2} \times[0,2]=1$. Then $g=h \mid S^{2} \times[0,2]$ is the required map.

4. Proof of Theorem 1. Let $\Sigma \in \delta$. We use the notation of $\$ 2$; hence, $\Gamma(\Sigma)=S \cup\left(\cup a_{x}\right)$ and $\Psi\left(S \cup\left(\cup a_{x}\right)\right)=d(S)$. Let $k$ be a map of $d\left(S^{3}\right)$ onto itself such that $k\left(d g\left(S^{2} \times[0,1]\right)\right)=d(S)$ and $k \mid d\left(S^{3}\right)$ $-d g\left(S^{2} \times[0,1]\right)$ is a homeomorphism onto $d\left(S^{3}\right)-d\left(S^{2}\right)$. This is possible since a manifold with boundary plus a closed collar is canonically homeomorphic to the manifold with boundary. Define $f: S^{3} \rightarrow \rightarrow d\left(S^{3}\right)$ by

$$
\begin{aligned}
f(x) & =k d h_{0}(x), & & x \in C_{0}, \\
& =k d h_{1}(x), & & x \in C_{\mathbf{1}} .
\end{aligned}
$$


Evidently $f$ is a homeomorphism and $f(\Sigma)=d(S)$. Thus $\Sigma$ is equivalent to $d(S)=\Psi \Gamma(\Sigma)$ and $\Psi_{*} \Gamma_{*}=1$.

Let $S \cup\left(\cup a_{x}\right) \in Q$. By Theorem 2 there is a homeomorphism $h: S \times[-1,2] \rightarrow S^{3}$ such that $h\left(y, \frac{1}{2}\right)=y$ for each $y \in S$ and $h(x \times[-1,2]) \supset a_{x} \cap h(S \times[-1,2])$ for each $x \in X$. Let $f_{1}$ be a map of $S^{3}$ onto itself such that

$f_{1} \mid S^{3}-h(S \times[-1,2])=1, \quad f_{1}(h(y \times[1,2]))=h(y \times[-1,2])$

and

$$
f_{1}(h(y \times[0,1]))=h\left(y, \frac{1}{2}\right)
$$

for each $y \in S$. By Theorem 3 there is a map $f_{2}$ of $S^{3}$ onto itself such that $f_{2}$ maps each component of $a_{x}-h(x \times[0,1])$ to a point for each $x \in X, f_{2}$ is a homeomorphism elsewhere, $f_{2}\left(a_{x}\right)=a_{x}$ and $f_{2} \mid S=1$. It follows from property (6) that there is a map $f_{3}$ of $S^{3}$ onto itself such that $f_{3}\left(f_{2}(h(y \times[0,1]))\right)$ is a point for all $y \in S$ and $f_{3}$ is a homeomorphism elsewhere. Then $f=f_{3} f_{2} f_{1}^{-1}$ is a map of $S^{3}$ onto itself such that $f\left(a_{x}\right)$ is a point for each $x \in X$ and $f \mid S^{3}-\bigcup a_{x}$ is a homeomorphism. Then $\Sigma=f(S) \in \Psi_{*}\left(S \cup\left(\cup a_{x}\right)\right)$.

Let $C_{0}$ and $C_{1}$ be the closed complementary domains of $\Sigma$ in $S^{3}$. Define an imbedding $h_{i}: C_{i} \rightarrow S^{3}$ such that $f_{3} h_{i}: C_{i} \rightarrow C_{i}$ is the identity. Define an imbedding $g: S \times[0,1] \rightarrow S^{3}$ by $g(y, t)=f_{2} h(y, t)$. Then $g(S \times i)=h_{i}(\Sigma)$,

$$
h_{0}^{-1} g(y, 0)=f_{3} f_{2} h(y, 0)=f_{3} f_{2} h(y, 1)=h_{1}^{-1} g(y, 1)
$$

and $g\left(y, \frac{1}{2}\right)=f_{2} h\left(y, \frac{1}{2}\right)=f_{2}(y)=y$. From this it follows that $S \cup\left(\cup a_{x}\right)$ $\in \Gamma_{*}(\Sigma)$ and hence $\Gamma_{*} \Psi_{*}=1$.

5. Let $\delta_{1}$ be the collection of 2 -spheres in $S^{3}$ each of which is locally flat except at a countable number of points and let $Q_{1}$ be the collection of spheres with arcs in $S^{3}$ such that the collection of arcs is countable. By Theorem 1 of [2] each set in $Q_{1}$ satisfies property (6).

THEOREM 4. The duality between $s$ and a restricts to a duality between $S_{1}$ and $Q_{1}$.

Let $S_{2}$ be the collection of 2 -spheres in $S^{3}$ each of which has a closed complementary domain which is a closed 3-cell and let $a_{2}$ be the collection of spheres with arcs in $S^{3}$ such that all the arcs are contained in one of the closed complementary domains of the tame sphere. By Theorem 3 each set in $a_{2}$ satisfies property (6). 
THEOREM 5. The duality between $S$ and a restricts to a duality between $S_{2}$ and $a_{2}$.

\section{REFERENCES}

1. B. J. Ball, The sum of two solid horned spheres, Ann. of Math. (2) 69 (1959), 253-257. MR 21 \#321.

2. R. H. Bing, Upper semicontinuous decompositions of $E^{3}$, Ann. of Math. (2) 65 (1957), 363-374. MR 19, 1187.

3. R. H. Fox and E. Artin, Some wild cells and spheres in 3-dimensional space, Ann. of Math. (2) 49 (1948), 979-990. MR 10, 317.

4. N. Hosay, The sum of a cube and a crumpled cube is $S^{3}$, Abstract \#607-17, Notices Amer. Math. Soc. 10 (1963), 666.

5. L. L. Lininger, Some results on crumpled cubes, Trans. Amer. Math. Soc. 118 (1965), 534-549. MR 31 \#2717.

6. Joseph Martin, The sum of two crumpled cubes, Michigan Math. J. 13 (1966), 147-151. MR 32 \#8324.

7. C. D. Sikkema, A duality between certain spheres and arcs in $S^{3}$, Trans. Amer. Math. Soc. 122 (1966), 399-415. MR 33 \#7991.

Florida State University, Tallahassee, Florida 32306 